\title{
Digital Spatial Models in Technology as a Development Tool of the Intellectual Creative Aspect within Education
}

\author{
Rushana R. Anamova, Svetlana A. Leonova, \\ Lidiya G. Nartova, Victor P. Tereshchenko \\ Moscow Aviation Institute (MAI), 125993, Volokolamskoe highway, 4, Moscow, Russia
}

\begin{abstract}
This is a conceptual approach to knowledge acquisition at technical universities. The emphasis is on the consistent possession of knowledge: the acquisition of new and preservation of the existing ones. At the same time, the grapho-geometric component in technical education is one of the most important elements of fundamental (general engineering) education. It is carried out at the initial stage in the process of studying technical disciplines included in the relevant curricula. Applied geometry plays an important role in this process, and it includes such directions as descriptive geometry, analytic geometry, engineering graphics, and computer graphics that provide spatial interpretation of geometric objects and display them on a plane. This combination of subjects studied significantly enriches the geometric development within student's intellectual world.
\end{abstract}

Keywords - grapho-geometric component, applied geometry, intellectual development, creative imagination, feature of second-order rotation surfaces.

\section{Introduction}

The disciplines related to the direction "Applied Geometry" includes such sections as descriptive geometry, analytic geometry, and computer graphics

DOI: $10.18421 /$ TEM93-45

https://doi.org/10.18421/TEM93-45

Corresponding author: Rushana R. Anamova, Moscow Aviation Institute (MAI), Moscow, Russia.

Email: svetlana.a.leonova@mail.ru

Received: 23 March 2020.

Revised: 03 July 2020.

Accepted: 12 July 2020.

Published: 28 August 2020.

(cc)BY-NC-ND(C) 2020 Rushana R. Anamova et al; published by UIKTEN. This work is licensed under the Creative Commons Attribution-NonCommercial-NoDerivs 4.0 License.

The article is published with Open Access at www.temjournal.com that implements visualization and processing of spatial objects using electronic computer technology. A wide range of proposed systems of solid-state modeling allows solving complex problems of geometric modeling of technical surfaces [1], [2].

In this regard, it should be noted, in the broadest sense, that today we are dealing with a student, and tomorrow it is an engineer, designer, researcher, or scientist. Accordingly, there are tasks arising from this, facing the developers of curricula forming a sequence of disciplines, which include technical training of specialists, in particular, in the aviation industry [3]. The consistent mastery of fundamental knowledge such as descriptive geometry, analytic geometry, and engineering graphics, combined with a practical course in computer capabilities is obvious [4], [5]. As a result of the sequence of acquired knowledge during the curricula, the student comes to understand the physical meaning of their actions, which contributes to the acquisition of skills in designing aviation products already at the stage of general engineering training [6].

2. Concept of the Formation of the Graphogeometric Component in the Technical Education Within Higher Educational Institutions

When studying the discipline "Descriptive Geometry", there are two clear main trends:

1) Introducing general issues related to the intellectualization of engineering education into the method of presenting educational material, such as:

- need for rigid structuring of general knowledge, basic concepts, which, as it is known, do not constitute a lot of simple attributes, but have a logically justified internal structure, held together by a body of knowledge;

- introduction of abstract principles regarding formalization, translation into harmonious 
algebraic structures, necessary for the study of subsequent courses in academic disciplines;

- selection of the most successful learning algorithms;

- widespread use of the principles of didactics, i.e. analogies, in particular, its two varieties:

○ for solving specific problems;

$\circ$ for prediction. For example, if the object of analogy is a certain system of axioms, then the knowledge that may be theorems is valid in this system.

2) Successful use of the content of visual geometry, effectively develop the spatial imagination among students, and it has a brilliant embodiment in classical geometry [7], [8].

All this makes it possible to use adequate geometric modeling, which allows comparing spatial geometric images (originals) and their planar equivalents using the classical G. Monge scheme and the apparatus of perspective geometry [9]. Since the course of descriptive geometry contains very complex geometric configurations, it is advisable to study them from different points of view and with the use of various research methods and expositions [10].

For example, the following working scheme of actions can be proposed: visual image of an object its analytical description - corresponding analysis - planar model.

Thus, unified system of geometric education, represented by the following cycles (in freshman classes) has to be in higher technical educational institutions:

- descriptive geometry with elements of analytic geometry;

- engineering graphics;

- computer graphics;

- geometric knowledge related to specialization in senior classes combined in constructive geometry.

Their content includes a parallel study of linear geometric images (points, lines, planes, and their incidents), which is a linear descriptive geometry and more complex geometric figures that are already included in curved descriptive geometry. Particular attention is paid to the similarity and difference of the studied objects and their interpretations in the process of synthetic and analytical methods of research.

Since the motivational foundations of training are not indifferent in the educational process, it is advisable to follow the principles of the functional purpose comprising geometric figures in senior classes, which will lead to the second cycle of training:
- higher engineering (specialized) geometry, based on the contents of the first cycle and including special geometric knowledge;

- fundamentals of geometric modeling and theoretical research of special surfaces.

It should be noted that the first semester of firstyear students is an extremely important stage in the development of knowledge. This is affected by the new specifics of studying at a university and its fundamental difference from school education. There is an increased possession of knowledge: acquisition of new and the preservation of existing ones. Knowledge is functional if it is involved in the process of productive thinking. This means that in the first half of the semester we need the most severe control over training, control in a wide variety of acceptable forms: systematic questioning, enhanced repetition of the previous material of the classes, verification of student's individual work, etc. This circumstance, even spontaneously, will lead to a rigid structuring of the studied material and its deeper assimilation. It is advisable to note that from the scientific and philosophical point of view, in the learning process, two main points or two of its main principles come across naturally: principle of acquisition and accumulation of knowledge (information), i.e. principle of possession, and the next is the deepest path of knowledge. This is the acquisition of the initial knowledge necessary for the second stage of training, the stage of productive creative knowledge (for more deeply knowledge), talent for applying it to solve specific problems now and in the future, future process of continuous selfeducation, i.e. necessary quality of the future specialist-intellectual. This "luxury" knowledge determines the position of a specialist in the future and hisher demand. It is advisable to note that the modern world is oversaturated with information; therefore academic and component selection should be made in all types of educational material presented [11].

As it was indicated, in the educational process in descriptive geometry that a special place is occupied by visual geometry and its methods, which have found brilliant embodiment in the classical methods of fine geometry (axonometric systems of different levels, their generalized theorems, Monge surfaces, etc.).

This fact makes it possible to use adequate geometric modeling, which allows the comparison of spatial geometric images and their planar equivalents in the classical schemes by G. Monge and axonometry. 


\section{Representation Features of Geometric Images}

After considering the elements of the concept within general geometric education, it is advisable to go on to study the representation features of specific geometric images that contribute to the development of a creative intellectual aspect in the learning process: theory and practice of surface images, their features, and solving problems in incidents of various characters.

In accordance with the general principle of descriptive geometry, a surface as a certain geometric image can be defined graphically and analytically.

In the first case of metric certainty, the concept of a surface determinant $\boldsymbol{F}$ should be introduced: it is a set of independent geometric elements (conditions, parameters) that uniquely determine the surface in space. Naturally, methods for determining surfaces have their own specific features. For example, we can assume that a surface is a continuous twoparameter (two-dimensional) set of points. Here, there is a natural connection with analytical geometry proposed in the 17th century by outstanding French scientist René Descartes, and leaning on a rectangular coordinate system. On the plane, these are two real numbers $x$ and $y$ : abscissa and ordinate. Using these coordinates, each point on the plane can be identified with a pair of real numbers $x$ and $y$. This circumstance allows expressing geometric figures by algebraic equations and algebraic equations as geometric figures, which makes it possible to more clearly clarify (visualize) geometric and algebraic concepts and properties of figures, as well as the relations between them.
Thus, geometric ideas become more abstract and, therefore, easily formulated. Algebraic ideas become more lively, visual, and accessible to intuitive perception. This short comment makes it possible to more clearly and comprehensively consider various ways of describing surfaces. So, in full accordance with the Cartesian coordinate system on the surface, its own curvilinear system and its curvilinear coordinates (parameters) $u, v$ are introduced.

This naturally implies another definition of a surface: it is a continuous one-parameter (onedimensional) set of lines connected by a certain law of formation. However, analytical, graphical, and kinematic methods for determining (setting) surfaces are also widespread in design practice.

\section{Representation Features of Geometric Images}

We select a surface region with curved coordinates $u, v$ applied to it. Now the current point $M^{i}$ is determined by its radius vector $O M^{i}$. In accordance with the general theory of vector algebra, we obtain the following equality:

Vector is the following:

$$
O M^{i}=i x(u, v)+j y(u, v)+k z(u, v),
$$

where the equalities are the following:

$$
x=x(u, v), y=y(u, v), z=z(u, v) .
$$

We determine the spatial coordinates of the current point $M$ on the surface $\Phi$ (Figure 1.), expressed in parametric form.

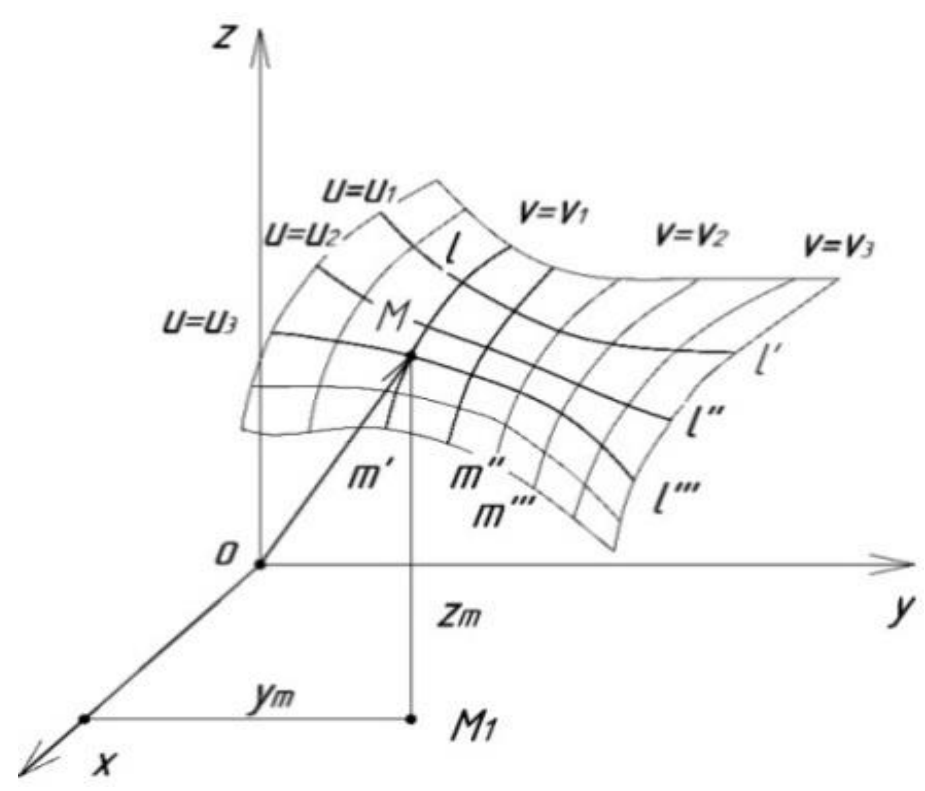

Figure 1. Curved surface network 
By excluding parameters $u$ and $v$ from the system (2) we can get the surface equation in the classic standard notation:

$$
z=f(x, y) \text {, or implicitly: } F=(x, y, z)=0 .
$$

Now the condition of membership (incidence) of a point $M^{o}$ has the following form: $F=\left(x_{0}, y_{0}, z_{0}\right)=0$. The surfaces specified in the analytical form have many structural representations depending on the properties of the function:

$$
F=(x, y, z) \text {. }
$$

So, if the function $F=(x, y, z)$ is polynomial of the degree " $n$ ", then the surface $\Phi$ defined by it called an algorithmic surface of the order $n$. The order of such a surface is defined as:

1) exponent of the defining polynomial;

2) number of intersection points of an arbitrary line with the surface (including imaginary points), i.e. points with minimal coordinates;

3 ) order of any plane curve on the surface under consideration.

As for analytical methods for defining surfaces, it is necessary to show students the possibility of their adaptation for computer implementation, without which any modern design is unthinkable. An example of such a "computer-oriented" geometry is the receptor (voxel in English terminology) method of geometric modeling, which effectively solves geometric problems of a certain class [12], [13]. We consider it very important to show the possibility of transition from graphical methods of representing objects used at the stage of creative comprehension to analytical ones, which allow determining with high accuracy their necessary engineering and geometric characteristics (position of the center of mass, trajectories of processing tools, etc.).

\section{Graphic Method for Specifying Surfaces}

According to the general method of display in descriptive geometry, the graphic method for determining the surface using projection-related areas (compartments) of surfaces is also widely used.

\section{Technical (kinematic) Method for Specifying Surfaces}

This method is based entirely on the basic principles of kinematic motion. Moreover, the systematization is based on the continuous movement of two interconnected lines in space, where the first line is a generator, and the second one, along which the movement is made. It is a directrix. One family of lines defines the law of motion for others. This allows the nature of the generator to classify the surface. For example, if the generator is straight, then the surface $\Phi$ is ruled; if the generator is a circle, then the surface $\Phi$ is cyclic. In turn, the law of motion of the generator ensures the existence of surfaces of revolution, parallel transfer, or skewed surface $\Phi$.

A continuous set of generators and directrices on the surface defines a curvilinear coordination network (Gaussian coordinates), similar to the Cartesian system in the plane. Based on linear transformations of elementary surfaces, we can obtain more complex surfaces of various classes, which are widely used in engineering. Here is an example. The surface of rotation is formed by the rotation of a line $(l)$ around a straight axis $(i=z)$. Each point of the generator describes a circle $p$ (parallel). All parallels are a one-parameter family of circles perpendicular to the common axis $i=z$. The axis of rotation is the geometric locus of the centers of all parallels. All parallels are similar (Figure 2.). Planes passing through the axis of the surface $\Phi$ cross it along the meridian $(m)$, moreover, all meridians are congruent, and the meridian belonging to the frontal axial plane of the level is called the main one and represents the outline of the surface of revolution.

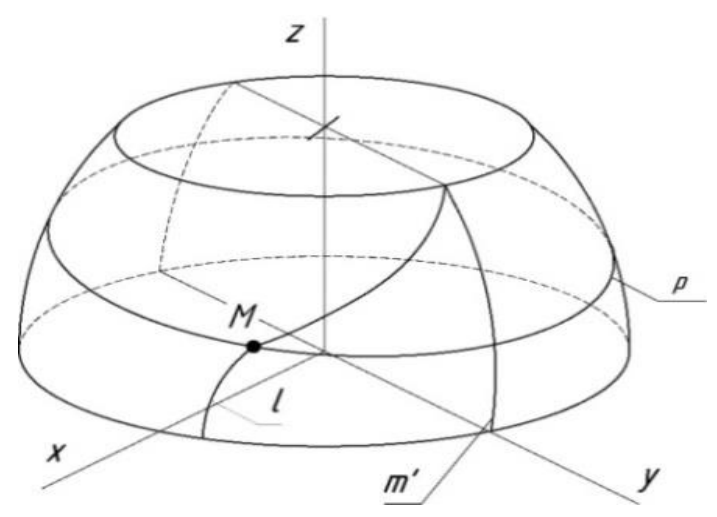

Figure 2. General surface of revolution 
There are the following main properties of the surface of revolution from the above reasoning:

1) Any secant axial plane is a plane of symmetry of the surface of revolution $\Phi_{0}$.

2) Generator can be formed only by a line from the surface, and it is having at least one common point with each parallel.

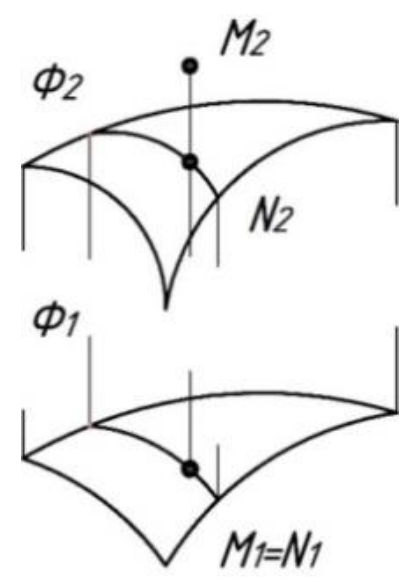

a)
3) Any planar section of a surface of revolution $\Phi$ is a curve, symmetric about the rectangular projection of the axis $z$ to the plane of symmetry $\Gamma_{o}^{\prime}$

Surface criterion $\Phi$ in space and on the epure consists in the possibility of a unique construction of the current point $M$ on it. There is the visual scheme for solving this problem in the Figure 3. $(a, b)$.

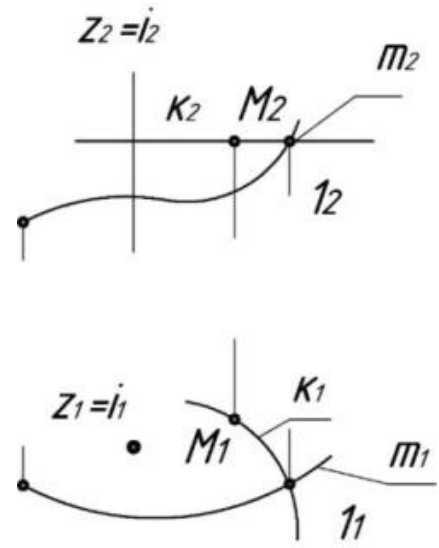

b)

Figure 3. Incidence of surface points

The scheme for obtaining the equation of the surface of revolution comprising the general form can be represented as follows (Figure 4.).

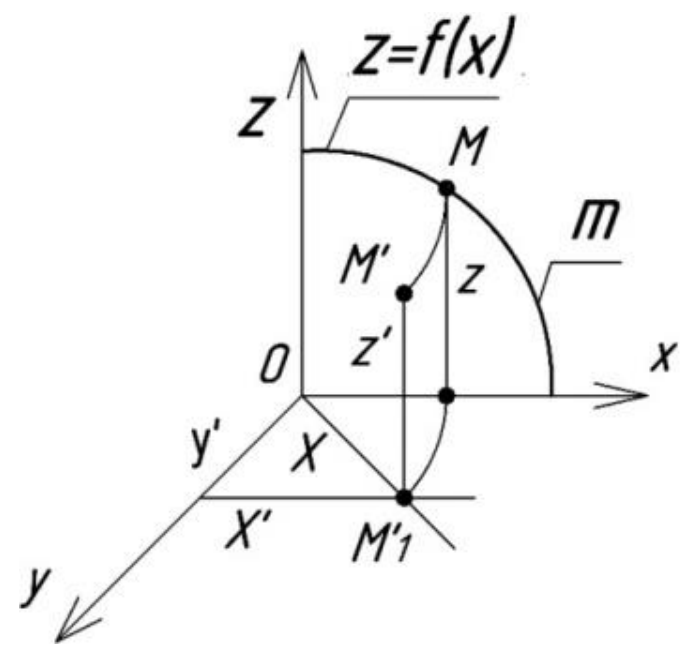

Figure 4. Derivation of the surface equation

If in the coordinate system $O x y z$ there is a meridian $\mathrm{m}$ of the future surface, its equation is the following: $z=f(x)$. Meridian rotates in the system $O x y z$ and current point $M$ took a position $M^{\prime}$. Point coordinates $M$ are $(x, o, z)$, and $\left(x^{\prime}, o^{\prime}, z^{\prime}\right)$ for $M^{\prime}$ respectively (Figure 4.).

Now

$$
O M_{1}{ }^{\prime}=O M_{1}=\mathrm{r}=\mathrm{x}=\sqrt{x^{\prime 2}+y^{\prime 2}} .
$$

Substituting the coordinate values into the equation of the initial meridian, we can write the equation of the desired surface $\Phi$ in the following form: $\mathrm{z}=\mathrm{f}\left(\sqrt{x^{2}+y^{2}}\right)$. This rule is valid for all surfaces of revolution of arbitrary and particular types.

If there is a surface equation, we can model the section lines of this surface by planes of different positions. To solve this problem, we use the section method. One of the most interesting and widely used in various fields of technology is selected surface of a one-sheeted hyperboloid of revolution defined by the canonical equation:

$$
\frac{x^{2}+y^{2}}{a^{2}}-\frac{z^{2}}{b^{2}}=1
$$


Additional interesting properties of this surface can be obtained by the method of sections. So the plane $\Gamma$ is parallel to the plane $O x y$, and $z= \pm h$. The result is a one-parameter family of circles (parallels) (Figure 5., Figure 6.).
If $z= \pm h$, equations of all sections has the following form: $a^{2}\left(b^{2}+h^{2}\right) / b^{2}=x^{2}+y$, where

$$
x^{2}+y^{2}=R^{2}=a^{2}\left(b^{2}+h^{2}\right) / b^{2} .
$$
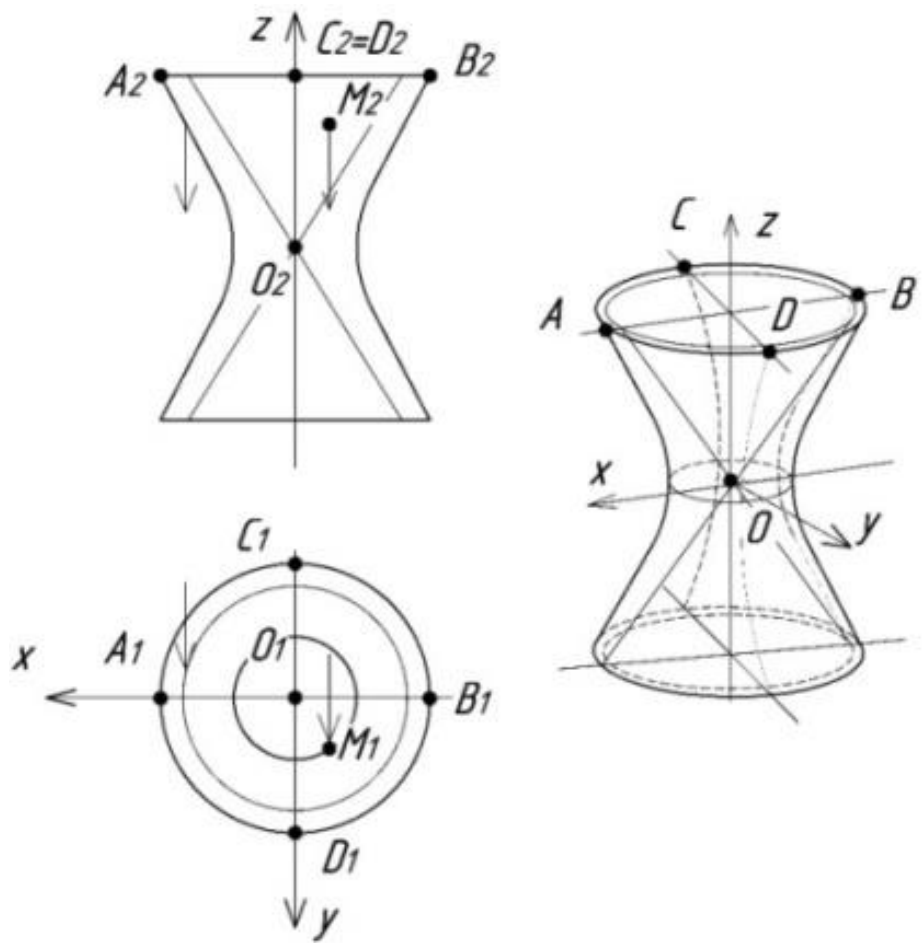

Figure 5. Sections of one-sheeted hyperboloid of revolution

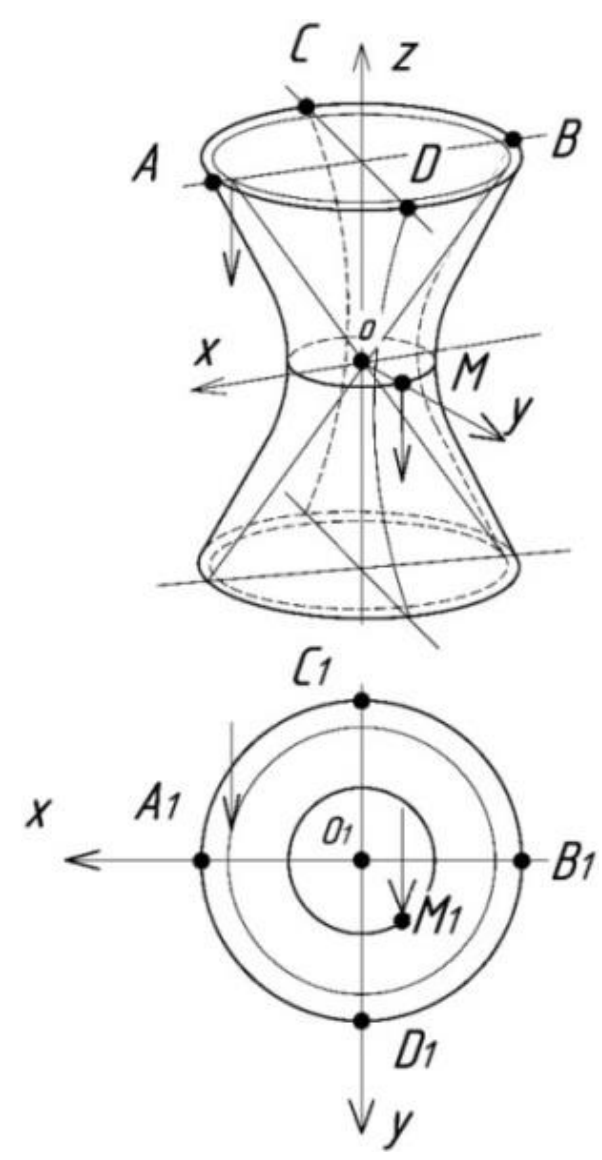

Figure 6. Surface visualization 
Next, let the secant planes be parallel Oxy. Their equation is $x= \pm l$, where the intersection lines are determined in three different cases: $y^{2} / a^{2}-z^{2} / b^{2}=\left(a^{2}\right.$ -

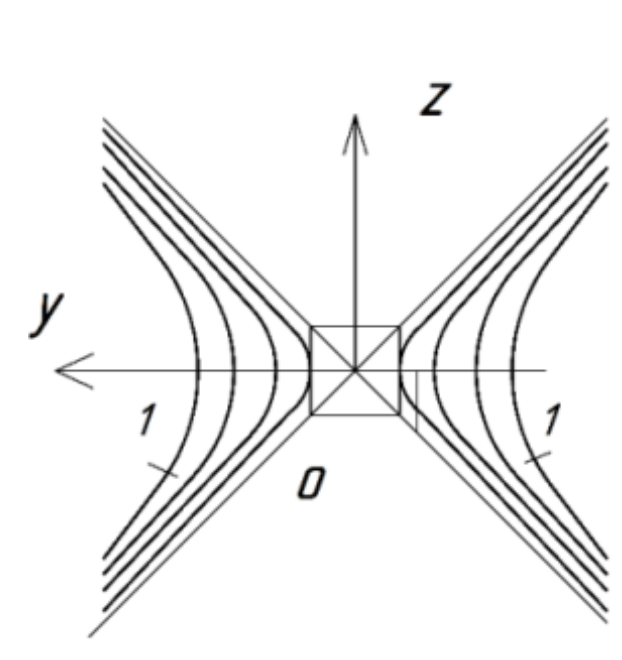

a) $\left.l^{2}\right) / a^{2}$, under the following conditions: $l^{2}>a^{2} ; l^{2}<a^{2}$ $; l^{2}=a^{2}$ (Figure 7. a, b).

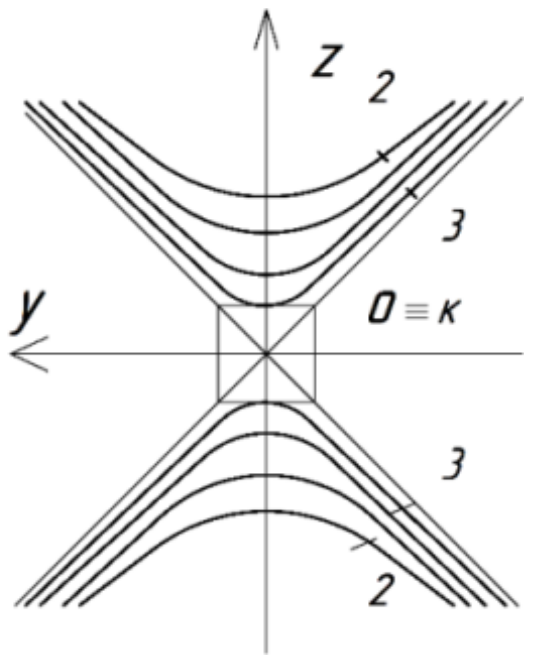

b)

Figure 7. (a, b). Sections of the surface of rotation

Figure 8. shows a framework of rectilinear surface generators of one-sheeted hyperboloid of revolution. One more feature of this surface should be noted.
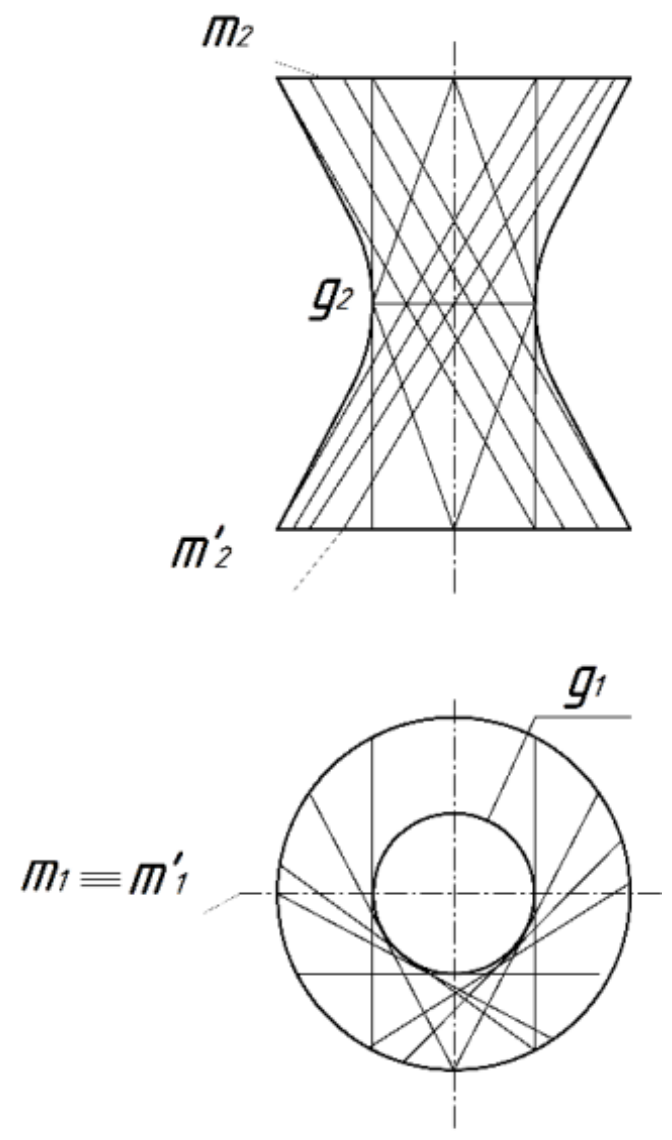

Figure 8. Frame of rectilinear surface generators of a one-sheeted hyperboloid of revolution 
There are two families of skew lines and a parallel of the smallest radius: neck (compression line or striction line of the surface: $g_{1}, g_{2}$ ) on it. Therefore, in the first case $\left(l^{2}>a^{2}\right)$, the secant planes cross the neck, and the surface, respectively, along the hyperbolas with the real axis $O y$. Similarly in the second case $\left(l^{2}<a^{2}\right)$ we get a family of hyperbolas with a real axis Oz. However, while designing, the third case has found the greatest application, when the cross section is straight lines $\left(l^{2}=a^{2}\right)$.

If $l^{2}=a^{2} ; x= \pm a$, we get the following system of equations:

$$
\left\{\begin{array}{l}
\frac{y}{a}+\frac{z}{b}=0 \\
\frac{y}{b}-\frac{z}{b}=0
\end{array} ;\right.
$$

The obtained equations determine two lines in space. Each of them in the process of rotation forms a family of lines, i.e. only two series of rectilinear generators (Figure 8.). As a visual application to these analytical calculations, an image of the surface is given in a visual form and on the Monge epure.

\section{Conclusion}

As a result, it should be noted that a consistent study including the visual and analytical characteristics of complex surfaces naturally contributes to a creative spatial imagination based on the theoretical foundations of applied surface geometry. Theoretical studies carried out in the article on the methods of surface images in the future will be a fundamental basis in the educational process in various sections of engineering graphics.

There is the concept that includes the formation of grapho-geometric knowledge at freshman classes of higher technical educational institutions in the form of a single system, which is represented by the following cycles:

- descriptive geometry with elements of analytic geometry;

- engineering graphics;

- computer graphics;

- geometric knowledge related to specialization in senior classes combined in constructive geometry.

There are examples of the representation features of specific geometric images that contribute to the development of a creative intellectual aspect in the learning process: theory and practice of surface images, their features, and solving problems in incidents of various characters.

In accordance with the general principle of descriptive geometry, a surface as a certain geometric image can be defined graphically and analytically.

The proposed concept was tested at Moscow Aviation Institute during the coursework of the discipline "Descriptive Geometry" in "Aircraft and Helicopter Engineering" and "Aircraft Engines" departments.

\section{References}

[1].Bol'shakov, V. P., Bochkov, A. L., \& Lyachek, Y. U. T. (2015). Solid modeling of parts in CAD systems: AutoCAD, KOMPAS-3D, SolidWorks, Inventor, Creo. Publisher, Piter.

[2].Yeltsov, M. Yu., Goncharov, P. A., Oseyuk, V. A., Laptev, I. V., \& Korshikov, S. B. (2016). NX for the mechanical engineer. Moscow: DMK press. [In Russian].

[3].Zharkov, N. V., \& Finkov, M. V. (2019). AutoCAD 2019. Complete Guide. St. Petersburg: Science and Technology. [In Russian]

[4].Anamova, RR, \& Nartova, LG (2019). Geometric spatial ability as an element of cognitive learning process. Periodico Tche Quimica , 16 (32), 542-550.

[5].Bodryshev, V. V., Babaytsev, A. V., \& Rabinskiy, L. N. (2019). Investigation of Processes of Deformation of Plastic Materials with the Help of Digital Image Processing. Periódico Tchê Química. 16(33), 865-876.

[6].Anamova, R. R., Leonova, S. A., Khvesyuk, T. M., \& Khotina, G. K. (2019). Systematic approach to the aircraft industry engineers preparation. Spravochnik. Inzhenernyi zhurnal, 49-56. [In Russian].

[7].Bairaktarova, D., Reyes, M., Nooshin Nassr, P. E., \& Carlton T. (2015). Spatial Skills Development of Engineering Students: Identifying Instructional Tools to Incorporate into Existing Curricula. 122nd ASEE Annual Conference \& Exposition. June 14-17. Seattle: WA.

[8].Berkowitz Biran, M., \& Stern, E. (2018). Which Cognitive Abilities Make the Difference? Predicting Academic Achievements in Advanced. Journal of Intelligence, 6(4), 48.

[9].Gold, A. U., Pendergast, P. M., Ormand, C. J., Budd, D. A., Stempien, J. A., Mueller, K. J., \& Kravitz, K. A. (2018). Spatial skills in undergraduate studentsInfluence of gender, motivation, academic training, and childhood play. Geosphere, 14(2), 668-683.

[10]. Gutierrez, J. C., Holladay, S. D., Arzi, B., Gomez, M., Pollard, R., Youngblood, P., \& Srivastava, S. (2018). Entry-level spatial and general non-verbal reasoning: Can these abilities be used as a predictor for anatomy performance in veterinary medical students?. Frontiers in veterinary science, 5, 226.

[11]. Hartatiana, H. (2018). Improving Junior High School Students' Spatial Reasoning Ability Through Model Eliciting Activities with Cabri 3D. International Education Studies, 11(01), 2-9.

[12]. Markin, L. V. (2018, November). Discrete geometric models in problems of automated assembling of objects. In IOP Conference Series: Materials Science and Engineering (Vol. 451, No. 1, p. 012124). IOP Publishing.

[13]. Markin, L. V. (2019). Receptor (Voxel) geometric models in the tasks of automated layout. Asia Life Sciences, 28(1), 125-138. 